\title{
DIJKSTRA'S ALGORITHM APPLIED TO 3D SKELETONIZATION OF THE CEREBRAL VASCULAR TREE: EVALUATION AND APPLICATION TO SYMBOLIC DESCRIPTION
}

\author{
Verscheure L. ${ }^{1,2}$, Peyrodie L. ${ }^{2,3}$, Le Thuc V. ${ }^{1,4}$, Dewalle A.S. ${ }^{1}$, Maouche $S .{ }^{2}$, Vermandel M. ${ }^{1}$ \\ ${ }^{1}$ Inserm U703, 152 rue du Dr. Yersin, 59120 Loos, FRANCE \\ 2 LAGIS CNRS UMR 8146, USTL, 59655 Villeneuve d'Ascq, FRANCE \\ ${ }^{3}$ HEI, 13 rue de Toul, 59046 Lille Cedex, FRANCE \\ ${ }^{4}$ Neuroradiology Department, Salengro Hospital, Lille, France
}

\begin{abstract}
This paper describes and evaluates a method for cerebral vascular tree 3D skeletonization. This methodis based on the application of the minimum cost spanning tree using Dijkstra's algorithm. This method is especially appropriate to tubular objects. We briefly describe the steps of the method's general scheme, from the segmentation to the skeleton analysis, insisting on the chosen skeletonization method. Besides, we propose an original evaluation scheme of the method thanks to numerical and clinical phantoms. The final aim of this work is to provide a good synthetic representation of the vasculature which will be analyzed in order to describe the tree symbolically.
\end{abstract}

Index Terms - Medical imaging, vascular tree, angiography, 3D skeletonization, symbolic description

\section{INTRODUCTION}

Human physiology presents a big variety of elements following a tree structure (vascular systems, bronchial system, breast ductal network). These structures introduce a parent-child relationships scheme respecting a simple principle of organization into a hierarchy of elements: parents branches - children branches. The interest of the study of these structures in terms of symbolic description was already shown for different locations.

Since 1993, Gerig and al, [1] have proposed an extraction method of 3D structures to represent them by a symbolic approach where topological and geometric information is represented in form of a tree.

Later, in 2001, Bullit and al [2] underline the interest of the relationships knowledge between the different branches of cerebro-vascular tree-like aspect, in particular for their study in neurosurgery

More recently, Megalooikonomou and al [3] introduced a method to characterize, classify and analyse the similarities of tree structures in medical pictures. This study is applied to tree-like aspects of breast ductal network extracted from clinical data acquired by X-rays galactograms.

In a general way, the applications of symbolic description are numerous. Palagyi and al underline the importance of the field of applications (virtual navigation, complex structures exploration, tubular objects quantitative analysis...) in preamble of their article [4]

The purpose of this paper is to describe and evaluate a 3D skeletonization method based on the construction of the minimum-cost spanning tree thanks to Dijktra's algorithm [5]. This method is applied to the cerebral vasculature.

\section{METHOD}

The implementation of symbolic description often respects the same main scheme where description is acquired after an extraction of data (binarisation) and a skeletonization $[4 ; 5]$.

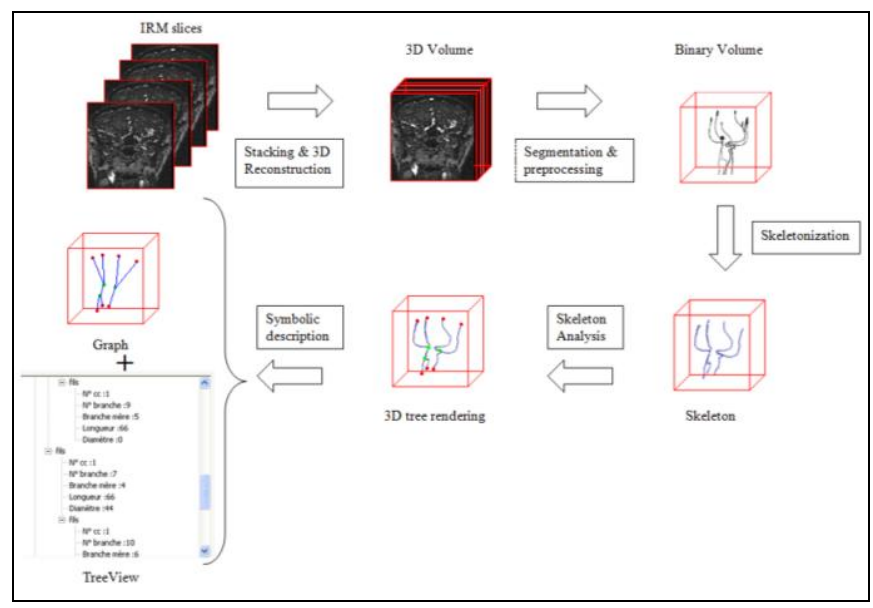

Figure 1 : Global scheme of the method

However, independently of the location and application, the problem remains skeletonization. Literature treating skeletonization is abounding so it would be tiring to do an 
exhaustive presentation. In this article, we focus on a method based on Dijkstra's algorithm, particularly used in coloscopy.

Consequently, our method respects the general scheme accepted in literature to skeletonise and then to extract the representation in graph of the tree: data segmentation, segmented data corrections, skeletonization of the segmented data, skeleton's branches and nodes identification and graph representation of the tree structure (Figure 1).

In this section, we present these procedures, with more emphasis on the skeletonization method.

\subsection{Segmentation}

The segmentation's solution used to extract the vascularization of pictures was represented in [7-9]. This algorithm is particularly interesting in our case since it allows segmenting independently angiographic pictures by IRM with or without injection of contrast fluid.

Methodology rests on the analysis of pictures of the Maximum of Intensity Projection (MIP). For every voxel, a degree of membership is calculated by inserting at the same time the grey level of its projection on MIP and Contrast to Noise Ratio (CNR) of initial images (Figure 2).

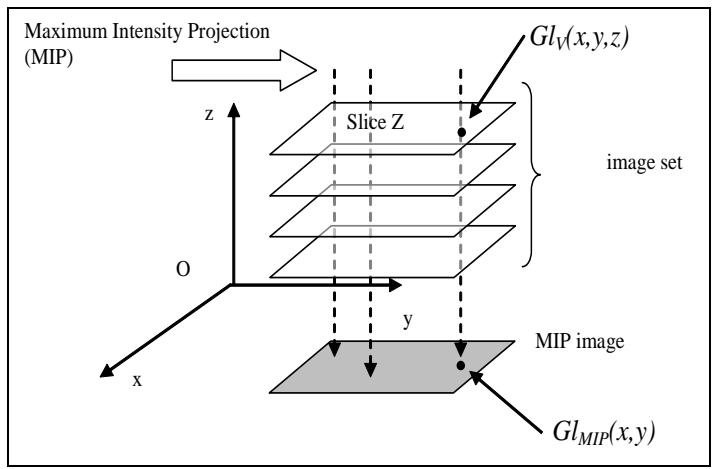

Figure 2 : Description of the algorithm based on MIP. GIV $(x, y, z)$ is the grey level in position $(x, y, z)$ of the $Z$ slice and GIMIP $(x, y)$ is the grey level of the projection of the voxel $(x, y, z)$ on MIP. Both values are combined to determine the membership of the voxel in the vascular tree.

\subsection{Skeletonization method based on Dijktra's tree construction algorithm}

This method is based on the use of the minimum cost path Dijkstra's algorithm. This skeletonization's solution introduced by Wan and al [5] was also used within the context of vascular aneurysms analysis $[10 ; 11]$.

However, in their articles, Hassan and al use a skeletonization's step for branches holding aneurysm only with a view to accomplishing Computed Fluid Dynamic simulations.
In this paper, we generalize the implementation of the algorithm in order to skeletonise the tree up to the most distal vessels.

In a general way, our skeletonization's method is based on two stages: minimum cost spanning tree construction (Dijkstra's algorithm) and then, centerline and attached branches extraction from Dijkstra's tree.

\subsubsection{Minimum cost spanning tree construction}

This step allows to draw the neighbourhood's links between every voxel and to allocate for each voxel a link towards his closest neighbour.

The minimum cost spanning tree requires a preliminary conversion of the volume into a 3D weighted graph. So, every voxel represents a node of graph and branches represent the relations of 6-neighbourhood between voxels.

Weight allocated for each node is the opposite of distance from the boundary (1/DFB).

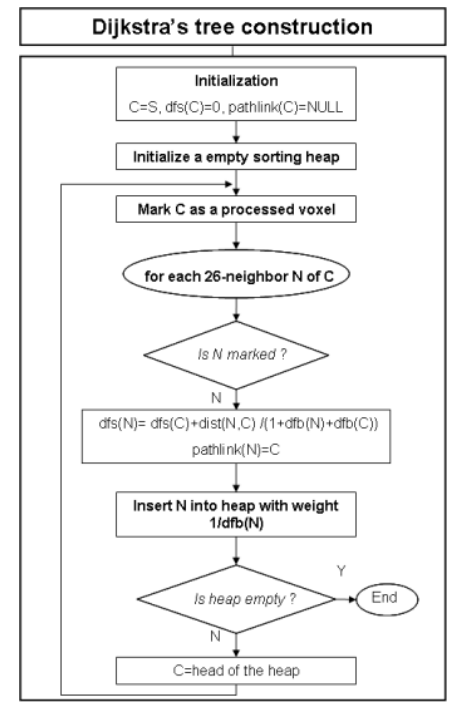

Figure 3 : Dijkstra's tree construction algorithm where the main steps are a) mark the source point $S$ (mark $(s)=$ true), define it as current node $(\mathrm{C}=\mathrm{S})$, put the pathlink at NULL, and the Distance From Source (DFS) at 0; b) Push the Ni neighbours of the $\mathrm{C}$ node if mark (Ni ) $=$ false, and initialize pathlink $(\mathrm{Ni})=\mathrm{C}$, calculate their distance from $\mathrm{S}$ node DFS(Ni ) =DFS (C) + dist (Ni, C) where dist is Euclidean distance;c) Pull the head of pile to treat a new node in $b$ )

\subsubsection{Centerline and attached branches extraction from Dijkstra's tree}

The algorithm of extraction of the centerline is based on scanning the tree from the node that has the maximum DFS. If we consider the path link attribute of each node and the tree to be an orientated graph (impossible step backwards), the centerline is defined as the longest branch.

Obviously, this step only allows the centreline extraction. In our perspective to generalize implementation, 


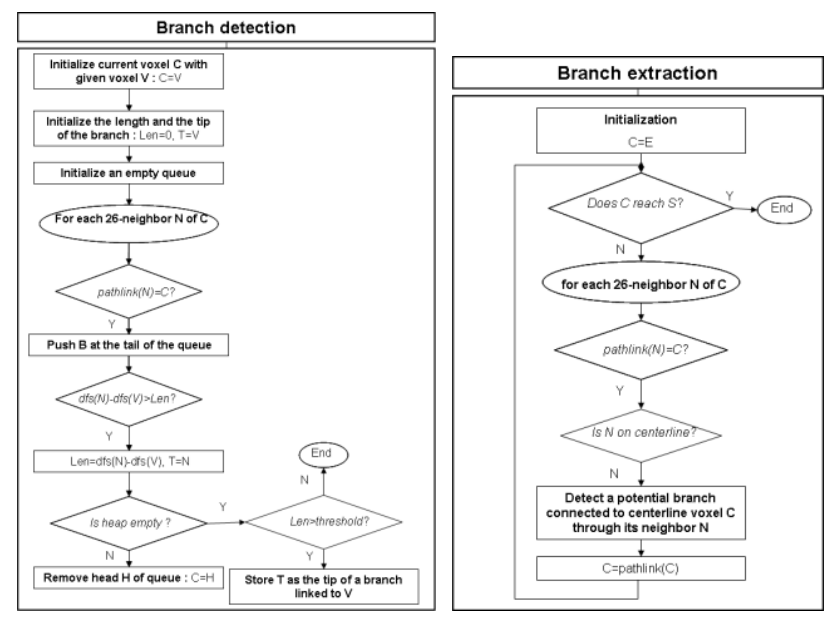

Figure 4 : Flowchart of branches extraction from Dijkstra's tree. «Branch extraction » calls "Branch detection» to detect branches linked indirectly to the current voxel.

it is necessary to iterate the process for every branch linked to the centerline. So, from the acquired centerline, [6] proposed the algorithm Figure 4. This method allows the extraction of the first generation branches.

To allow an extraction of the most distal branches, we chose to implement this algorithm in a recursive way. For each detected branch, we search its attached branches. This detection operates in the same way by scanning the branch but this time the criterion of conservation of the branch is not any more its distance from the source but its length.

\subsection{Symbolic description}

After the skeletonization of the 3D volume, the identification of different branches is traditionally done thanks to the analysis of voxels belonging to the skeleton. The detection of final voxels and junctions allows then to define every branch or vascular segment.

At this stage of the process, we are therefore capable of representing the vascular tree in the form of an orientated graph. A structural presentation of the tree then easily occurs where the object "branch" contains different information: the hierarchy of the branch (index in the list of branches), the list of voxels linked to the branch, its particular voxels (voxels terminals and junctions), its relations of filiations (rising and downward branches) and its ray (determined thanks to distance from the boundary of the voxels of the branch).

Figure 5 shows a tree-view representation of the result of the symbolic description.

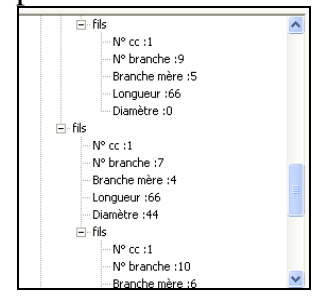

Figure 5 : Symbolic Description as Tree View including information about the different detected branches: connected component number, branch number, length, parent-child relationships and diameter.

\section{EXPERIMENTS AND RESULTS}

\subsection{Evaluation}

There are few methods of skeletonization algorithms evaluation. Palagyi and al [4] offer an interesting scheme for quantitative evaluation. From a physical ghost, a numerical ghost and in-vivo acquisitions, their plan of experience allows proving the number of discerned branches, the error of positioning, diameters, length and volumes of the discerned branches.

In our study, we adopted approximately the same strategy of evaluation. At first we worked out an interface in Matlab allowing generating numerical models from a reference skeleton. From a main branch, additional branches are linked forming the skeleton of a tree-like aspect. A volume is then developed from the skeleton and the given diameters. The numerical constituted model allows us to know the characteristics of the studied skeleton precisely.

Our study also concerned an anthropomorphic cerebral vascularisation phantom. Elastrat phantom (Shelley Medical imaging Technologies), consists of a model of the anterior and posterior circulation linked to a model of aneurysm on the communicating anterior artery. Pictures could be acquired in IRM (IRM Vision 1.5 T (Siemens, Erlangen, Germany) to assess algorithm in the real conditions of acquisitions.

For every volume, and to assess the robustness of the algorithm, as recommended in Palagyi and al [4], a rotation of $5^{\circ}$ from $-15^{\circ}$ to $15^{\circ}$ is applied before processing.

Following quantitative measurements were chosen to evaluate algorithm from the numerical models:the number of discerned branches, the error of positioning calculated by a Hausdorff's distance between reference skeleton and acquired skeleton. The diameters, length and volumes of the discerned branches are estimated by the reconstruction of the volume from the data of symbolic description and from the skeleton thanks to the overlap rate calculation with the original volume. Concerning the anthropomorphic phantom, we only calculate the number of branches and overlap rate.

\subsection{Results}

Algorithms were implemented on the own laboratory software platform (ArtiMED) developed in Borland $\mathrm{C}++$. Manipulations were performed on a Personal Computer (processor: AMD 2.4 GHz-RAM: 2Go). 


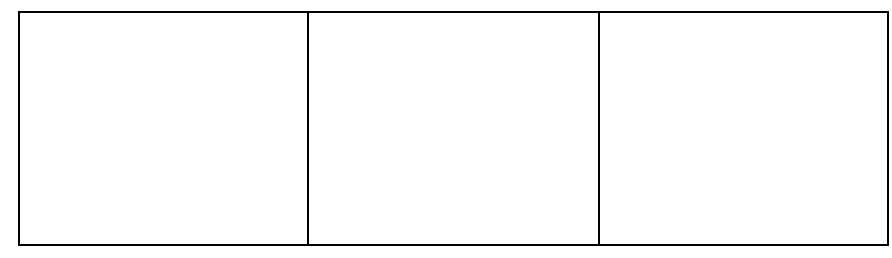

\begin{tabular}{|l|l|l|l|}
\hline & & & \\
\hline & & & \\
\hline & & & \\
\hline & & & \\
\hline & & & \\
\hline
\end{tabular}

\section{DISCUSSION AND CONCLUSION}

As displayed in the review [12], there are numerous 2D and 3D vessels extraction methods. The first difficulty is to find the most appropriate method in the studied case. For our tree-like structures study, we chose to approach the problem by skeletonization methods, particularly the Dijkstra's tree based method. It is necessary to underline the additional difficulty of working in 3D compared to the 2D methods.

The second problem encounteredduring our study is the lack of evaluation method for skeletonization algorithms. We offered a supplementary plan of experience of the quantitative evaluation scheme presented by Palagyi and al [4].

An analysis of the results acquired afterwards skeletonization by the method based on Dijkstra's algorithm allows us to conclude in the adequacy of method with treelike objects and more particularly in our case of vascular cerebral network.

Besides, this method allows, at the same time, calculating the skeleton and the maximum bowls radius. This property makes then possible the reconstruction of the initial volume without additional counting. The importance of this possibility of reconstruction is underlined during the stage of evaluation with the overlap rate calculation, allowing proving the ownership of reversibility of the skeleton.

However, we observed some limitations of this method particularly in the case of the cerebral vascular network. Indeed, like the majority of methods, our method is only semi-automatic since it imposes the choice of 2 thresholds during the extraction of the additional branches.

The second limitation is inherent to our application frame. Indeed, as Palagyi underlines, the result of the algorithm of Dijkstra is always a graph (without cycle). Now, we know the complex schematic form of this network which precisely introduces a cycle called Willis Polygon.

Concerning the symbolic description, the most remarkable advantage is the supplementary information contribution. Indeed, it can sometimes constitute an alternative in segmentation to establishing simulation intended models (flux calculation, surgery tools and vascular network interaction) to insert expert knowledge into software aided decision. Besides, this form of description seems to be quite adapted to nowadays enlarging CFD methods.

Finally, this symbolic description could bring clinical and methodological consequences notably in the field of neurosurgery with a direct application in the elaboration of a SEEG intervention planning software allowing discerning possible collisions between electrodes and cerebral vascular network.

\section{REFERENCES}

[1] G.Gerig, T.Kollera, G.Székely, C.Brechbuhler, and O.Kubler, "Symbolic descriptions of 3-D structures applied to cerebral vessel tree obtained from MR angiography volume data," Proceedings of 13th International Conference on Information Processing in Medical Imaging, IPMI'93, ser. Lecture Notes in Computer Science, vol. 687, pp. 94-111, 1993.

[2] E.Bullit, S.Aylward, K.Smith, S.Mukherji, M.Jiroutek, and K.Muller, "Symbolic description of intracerebral vessels segmented from Magnetic Resonance Angiograms and evaluation by comparison with X-Ray Angiograms," Medical Image Analysis, vol. 5, pp. 157-169, 2001.

[3] V.Megalooikonomou, M.Barnathan, D.Kontos, P.R.Bakic, and A.D.A.Maidment, "A representation and classification scheme for tree-like structures in medical images : analyzing the branching pattern of ductal trees in X-ray galactograms," IEEE Transactions on medical imaging, vol. 8, no. 4, pp. 487-793, Apr.2009.

[4] K.Palagyi, J.Tschirren E.A.Hoffman, and M.Sonka, "Quantitative analysis of pulmonary airway tree structures," Computers in Biology and Medicine, vol. 36, no. 9, pp. 974-996, Sept.2006.

[5] Z.Chen and S.Molloi, "Automatic 3D Vascular Tree Construction in CT angiography," Computerized Medical Imaging and Graphics, vol. 27, pp. 469-479, 2003.

[6] M.Wan, Z.Liang, Q.Ke, L.Hong, I.Bitter, and A.Kaufman, "Automatic Centerline Extraction for Virtual Colonoscopy," IEEE Transactions on medical imaging, vol. 21, no. 12, pp. 1450-1460, 2008.

[7] M.Vermandel, N.Betrouni, C.Taschner, C.Vasseur, and J.Rousseau, "From MIP image to MRA segmentation using fuzzy set theory," Computerized Medical Imaging and Graphics, vol. 31, no. 3, pp. 18-140, Apr.2007.

[8] M.Vermandel, AS Dewalle, P.Puech, C.Taschner, J.Rousseau, and N.Betrouni, "MRA segmentation algorithm using MIP and fuzzy set principles. Application to TOF contrast enhancement sequences," International journal of computer assisted radiology and surgery, p. -104, 2007.

[9] M.Vermandel, N.Betrouni, R.Viard, AS Dewalle, S.Blond, and J.Rousseau, "Combining MIP images and fuzzy set principles for vessels segmentation : application to TOF MRA and CE-MRA," International Conference of the IEEE Engineering in Medicine and Biology Society, pp. 6255-6258, 2007.

[10] T.Hassan, E.V.Timofeev, T.Saito, H.Shimizu, M.Ezura, Y.Matsumoto, K.Takayama, T.Tominaga, and A.Takahashi, "A proposed parent vessel geometry-based categorization of saccular intracranial aneurysms: computational flow dynamics analysis of the risk factors for lesion rupture.," American Journal of Neuroradiology, vol. 103, no. 4, pp. 662-680. Erratum in: J Neurosurg. 2005 Dec;103(6):1110., Oct.2005.

[11] T.Hassan, M.Ezura, EV.Timofeev, T.Tominaga, T.Saito, A.Takahashi, K.Takayama, and T.Yoshimoto, "Computational simulation of therapeutic parent artery occlusion to treat giant vertebrobasilar aneurysm." American Journal of Neuroradiology, vol. 25, no. 1, pp. 63-68, Jan.2004.

[12] C.Kirbas and F.Quek, "A review of vessel extraction techniques and algorithms," ACM Computing Surveys, vol. 36, no. 2, pp. 81-121, June2004. 